\title{
Exciton Condensation and Superfluidity in $\mathrm{TmSe}_{0.45} \mathrm{Te}_{0.55}$
}

\author{
P. Wachter \\ Laboratorium für Festkörperphysik, ETH Zürich, Zürich, Switzerland \\ Email: wachter@solid.phys.ethz.ch
}

How to cite this paper: Wachter, P. (2018) Exciton Condensation and Superfluidity in $\mathrm{TmSe}_{0.45} \mathrm{Te}_{0.55}$. Advances in Materials Physics and Chemistry, 8, 120-142.

https://doi.org/10.4236/ampc.2018.83009

Received: January 22, 2018

Accepted: March 19, 2018

Published: March 22, 2018

Copyright $\odot 2018$ by author and Scientific Research Publishing Inc. This work is licensed under the Creative Commons Attribution International License (CC BY 4.0).

http://creativecommons.org/licenses/by/4.0/

\begin{abstract}
In this publication details of the calculation of heat conductivity and thermal diffusivity, compressibility, sound velocity and exciton-polaron dispersion of $\mathrm{TmSe}_{0.45} \mathrm{Te}_{0.55}$ will be shown. The properties of excitons, coupling to phonons, producing thus polarons, but also blocking the phonons as running waves lead to an exciton condensation or exciton liquid. Surprisingly this exciton liquid is contained in a macroscopic crystal, a solid nevertheless, which becomes extremely hard due to the exciton liquid and finally exhibits a strange type of superfluid in a two fluid model, where the superfluid phase increases more and more below about $20 \mathrm{~K}$ until the whole exciton liquid becomes a superfluid at zero temperature. Never else a superfluid phase has been observed at such high temperatures.
\end{abstract}

\section{Keywords}

Exciton-Polarons, Superfluidity, Exciton Condensation, Heat Conductivity, Compressibility, Sound Velocity

\section{Introduction}

Excitons are electron-hole pairs and as such known in many materials, even in Si. Generally it is not easy to create so many excitons that they can interact with each other and finally can even condense in an exciton liquid. In standard experiments with laser pulses one can excite in semiconductors electrons from a valence band into a conduction band and then, due to the electron-hole attraction, the final state of the excited electron drops to somewhat below the bottom of the conduction band. An exciton is thus mobile, but it does not carry an electrical current due to its charge neutrality.

However, if one uses rare earth compounds, where the uppermost occupied state is a localized $4 \mathrm{f}$ state and the lowest conduction band is a $5 \mathrm{~d}$ band and one 
makes an optically induced $4 \mathrm{f}$ - $5 \mathrm{~d}$ transition, the hole in the $4 \mathrm{f}$ state will be localized and the electron just below the bottom of the conduction band will also be localized since it is bound by Coulomb attraction to its hole, the whole exciton will stay at the atom where the photo excitation occurred and it will not be mobile. Then this excitation energy will decay in typically $10^{-8} \mathrm{sec}$ with the emission of a photon or phonons at the same atom where it originated, resulting only in a localized excited atom in the lattice.

However a $\mathrm{p}^{6}-5 \mathrm{~d}$ exciton is always possible since the initial state is in a band. Thus Mitani and Koda [1] found Mott-Wannier excitons with thermo-reflectance in Eu chalcogenides with about $4 \mathrm{eV}$ (consult similar TmTe in Figure 1). The Tm mono-chalcogenides exhibit a metal-semiconductor transition inasmuch as $\mathrm{Tm}^{3+} \mathrm{S}^{2-}+\mathrm{e}$ is a trivalent metal with 1 free electron in the $5 \mathrm{~d}$ conduction band (Figure 1). The occupied $4 \mathrm{f}^{12}$ level is about $6.5 \mathrm{eV}$ below the Fermi energy $\mathrm{E}_{\mathrm{F}}$ and the empty $4 \mathrm{f}^{13}$ level is little above $\mathrm{E}_{\mathrm{F}}$. Experimental evidence comes mainly from $\mathrm{X}$-ray photoemission spectroscopy (XPS) and Bremsstrahlen isochromat spectroscopy (BIS) [2]. TmTe on the other hand is a divalent semiconductor $\mathrm{Tm}^{2+} \mathrm{Te}^{2-}$ with an occupied $4 \mathrm{f}^{13}$ level $0.3 \mathrm{eV}$ below the bottom of an empty $5 \mathrm{~d}$ band (Figure 1). The driving force behind this different character is the crystal field splitting of the $5 \mathrm{~d}$ band, which depends on the lattice constant due to the different anion radii.

TmSe, on the other hand, with an intermediate anion radius between sulphide and telluride has such a crystal field splitting of the $5 \mathrm{~d}$ band that the bottom of this band overlaps with the $4 \mathrm{f}^{13}$ level. This $\mathrm{f}$ - $\mathrm{d}$ hybridization on the one hand leads to some $\mathrm{d}$-character of the f-state and as a consequence to a narrow f-band and on the other hand to some f-character of the bottom of the d-band. It has been conventional to describe the new hybridized f-state as $4 \mathrm{f}^{13}-4 \mathrm{f}^{12} 5 \mathrm{~d}$, consisting of a quantum mechanically mixed state [3]. This new phenomenon is called intermediate valence, since the valence of rare earth ions is defined by the

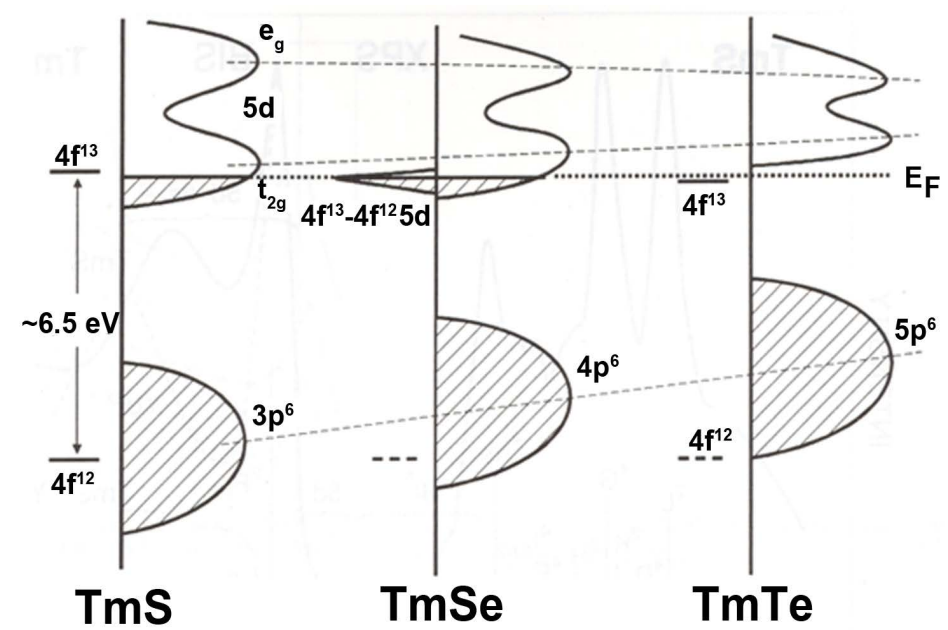

Figure 1. Electronic structure and density of states of the Tm chalcogenides, normalized to the Fermi level $\mathrm{E}_{\mathrm{F}}$. 
occupation of the f-state and thus TmSe has a valence, between $3^{+}$and $2^{+}$, in fact $2.85^{+}$. This can only be achieved if the $4 \mathrm{f}$-state is a narrow, band, which is partially filled with electrons [3]. But also in the $5 \mathrm{~d}$ band there are some free electrons, which yield in the visible a coupled plasma resonance of these electrons and are responsible for the copper-like colour of the crystals.

\section{Material Tailoring}

One can now make mixtures between the semiconducting TmTe and the metallic intermediate valence TmSe and thus tune the energy gap $\Delta \mathrm{E}$ between 300 $\mathrm{meV}$ and zero (metal) [3] [4]. Experimentally semiconducting $\operatorname{TmSe}_{1-\mathrm{x}} \mathrm{Te}_{\mathrm{x}}$ has been created with $\mathrm{x}=0.40,0.55,0.68$, corresponding to $\Delta \mathrm{E}$ of $40 \mathrm{meV}, 110-120$ $\mathrm{meV}, 170 \mathrm{meV}$. For these compositions the $\mathrm{f}$-state is so close to the $5 \mathrm{~d}$ band that some hybridisation occurs between the tails of the wave functions. We have the unexpected situation of intermediate valence semiconductors. This in turn means that the originally localized $4 \mathrm{f}^{13}$-state acquires now some bandwidth in the order of tens of meV.

Concerning now the existence of $4 \mathrm{f}-5 \mathrm{~d}$ excitons, we have created a situation where the hole state in the $4 \mathrm{f}$ is mobile and the electron is in a $5 \mathrm{~d}$ state below the bottom of the $5 \mathrm{~d}$ band. Now we have the possibility of $4 \mathrm{f}-5 \mathrm{~d}$ excitons. Of course the hole in a narrow $4 \mathrm{f}$-band has a large effective mass, so that the $4 \mathrm{f}-5 \mathrm{~d}$ exciton is a heavy, $\left(\mathrm{m}_{\mathrm{h}} \approx 50 \mathrm{~m}_{\mathrm{e}}\right)$, nearly immobile particle. These excitons without application of external pressure have a low concentration at low temperatures because thermal excitations into the excitonic state are rare.

It is thus the first time that an intermediate valence rare earth material, a $\mathrm{TmSe}_{1-\mathrm{x}} \mathrm{Te}_{\mathrm{x}}$ alloy, is used to create an enormous amount of excitons, which finally under high pressure condense and result in superfluidity.

From Bohr's formula for the hydrogen radius $\mathrm{a}_{\mathrm{H}}=0.53 \varepsilon \AA$ and from Figure 2 with the reflectivity for $\omega \rightarrow 0=50 \%$ we obtain $\mathrm{n}=\frac{1+\sqrt{R}}{1-\sqrt{R}}=5.8=\sqrt{\varepsilon}$. So the static dielectric constant $\varepsilon=34$ and the radius of the orbit of the exciton is about $18 \AA$. This would be a Mott-Wannier exciton. Its binding energy from the optical result is theoretically $\mathrm{E}_{\mathrm{B}}=13.6 / \mathcal{E}^{2}=15 \mathrm{meV}$, about the same as from the electrical measurement [4], but experimentally from Figure $2 \mathrm{E}_{\mathrm{B}} \approx 60 \mathrm{meV}$. The most complete measurements have been performed on $\mathrm{TmSe}_{0.45} \mathrm{Te}_{0.55}$ with an energy gap $\Delta \mathrm{E}$ of $110-120 \mathrm{meV}$, confirmed by far infrared reflectivity (see inset of Figure 2). Similar absorptive peaks as for $\mathrm{TmSe}_{0.45} \mathrm{Te}_{0.55}$ are absolutely missing in other divalent rare earth chalcogenides with only localized 4f-states (Figure 3).

In the fcc rocksalt structure the $4 \mathrm{f}^{13}$-band has a maximum at the $\Gamma$ point of the Brillouin zone and a minimum at the $\mathrm{X}$ point. The $5 \mathrm{~d}$ band dispersion has its minimum at the $\mathrm{X}$ point. An optical transition between the maximum of $4 \mathrm{f}$ and the minimum of $5 \mathrm{~d}$ would be an indirect transition and requires maximal the assistance of a $\Gamma-\mathrm{X}$ phonon for $\mathrm{k}$ conservation (black curve). 


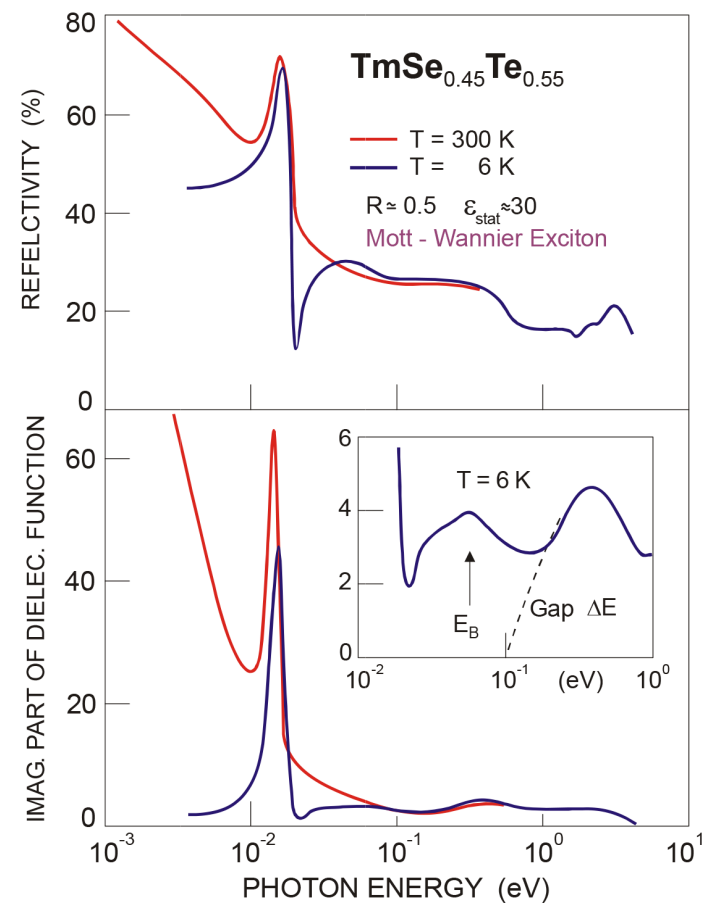

Figure 2. Reflectivity of $\mathrm{TmSe}_{0.45} \mathrm{Te}_{0.55}$ between $1 \mathrm{meV}$ and $6 \mathrm{eV}$ photon energy. At low temperatures the transverse optical (TO) phonons are the dominant feature. The inset in Figure 2 shows the absorptive part of the dielectric function and the energy gap $\Delta \mathrm{E} \approx 110 \mathrm{meV}$ and the binding energy of an exciton is $\mathrm{E}_{\mathrm{B}} \approx 60 \mathrm{meV}$ [5].

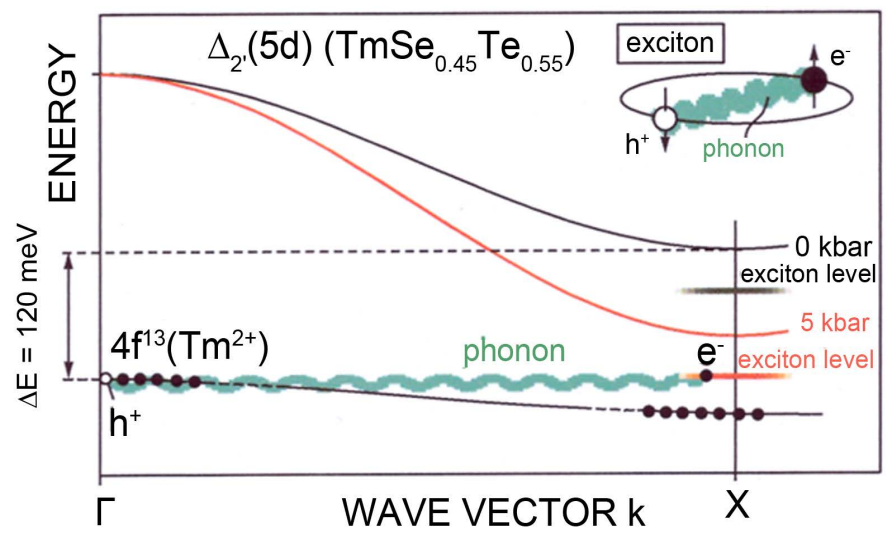

Figure 3. Schematic band structure of $\mathrm{TmSe}_{0.45} \mathrm{Te}_{0.55}$. Due to $4 \mathrm{f}-5 \mathrm{~d}$ hybridization the $4 \mathrm{f}^{13}$-state becomes a narrow band and has a dispersion. The exciton level with binding energy $\mathrm{E}_{\mathrm{B}}$ is indicated below the bottom of the $5 \mathrm{~d}$ conduction band (black curve). The red curve represents the band structure at $5 \mathrm{kbar}$ with the exciton level at $\mathrm{X}$ at the same height as the $4 \mathrm{f}-$ level at $\Gamma$. In green is the $\Gamma-\mathrm{X}$ phonon [6].

\section{Creation of Excitons}

Under hydrostatic pressure the bottom of the $5 \mathrm{~d}$ band at $\mathrm{X}$ with its exciton level will be lowered with respect to its center of gravity $\left(5 \mathrm{dt}_{2 \mathrm{~g}}-5 \mathrm{de}_{\mathrm{g}}\right)$ and shown for 5 
kbar the exciton level is exactly at the energy of the $4 \mathrm{f}$-state at $\Gamma$ (red curve). Now the highest energy electrons in the $4 \mathrm{f}^{13}$-band can spill without energy loss into the excitonic state at $\mathrm{X}$ leaving behind a positive hole. This transition needs the emission or absorption of $\Gamma-\mathrm{X}$ phonons which couple to the excitons. So in fact we are dealing with an exciton-polaron. With higher pressure the bottom of the $5 \mathrm{~d}$ band at $X$ will approach the energy of the $4 \mathrm{f}^{13}$-state at $\Gamma$ and the $4 \mathrm{f}$ electrons will enter directly the $5 \mathrm{~d}$ band and perform a first order semiconductor-metal transition.

In Figure 4 these transitions can be observed directly with resistivity in the isotherms versus pressure for $\mathrm{TmSe}_{0.45} \mathrm{Te}_{0.55}$. We look at first at room temperature $(300 \mathrm{~K})$ and find a classical pressure dependence of a resistivity, namely the resistivity of a semiconductor decreases with increasing pressure, because the energy gap $\Delta \mathrm{E}$ decreases with pressure and bands widen and finally the metallic state is achieved (above $11 \mathrm{kbar}$ ). Starting with about $5 \mathrm{kbar}$ and best observed at $5 \mathrm{~K}$ the resistivity now increases by about 3 orders of magnitude with pressure. This is exactly the pressure where excitons become stable states and electrons from the f-band, which have been thermally excited into the $5 \mathrm{~d}$ conduction band, drop into the excitonic state and are no longer available for electric conduction. We have created an excitonic insulator, a term coined by Sir Nevil Mott [7]. With further pressure increase the resistivity drops again, until now the $4 \mathrm{f}$ electrons can enter the $5 \mathrm{~d}$ band directly, which leads to a first order semiconductor-metal transition.

Here we want to make a remark of another possibility of the semiconductor-metal transition, namely a Mott transition to an electron-hole plasma or an electron-hole liquid. The experimentally derived exciton concentration is $3.9 \times$ $10^{21} \mathrm{~cm}^{-3}$ (see below). This is in fact too high (because of screening effects) for an

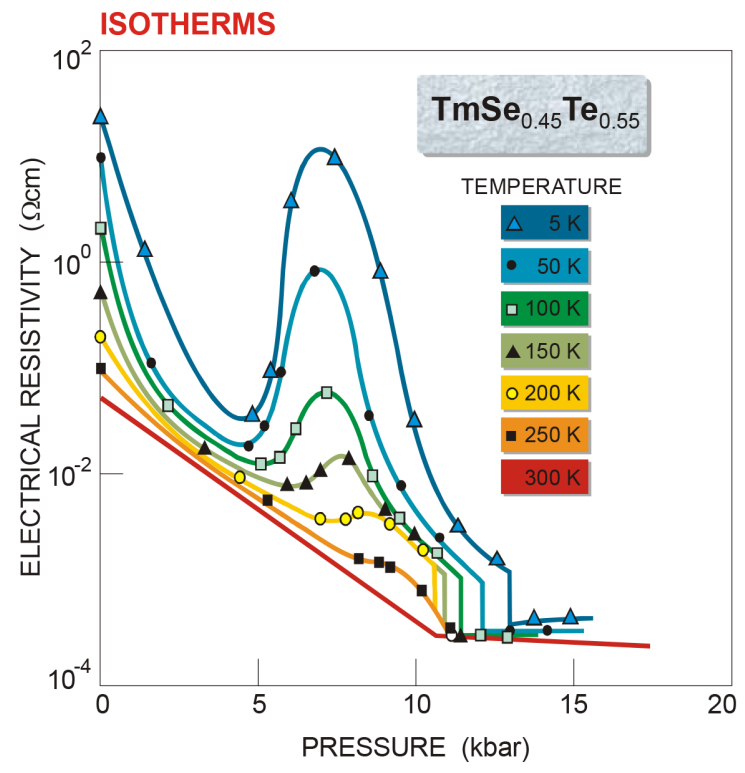

Figure 4. Isotherms of the electrical resistivity in $\mathrm{TmSe}_{0.45} \mathrm{Te}_{0.55}[5]$. 
electron-hole liquid as has been shown by Monnier et al. [8]. There it is calculated that the electron-hole liquid must be less than $10^{20} \mathrm{~cm}^{-3}$ excitons. In fact the rare earth nitrides may serve as experimental examples [9].

\section{Exciton Condensation}

Since for the exciton creation no energy is needed their number is enormous. But not all $4 \mathrm{f}$ electrons can form excitons, because as electric dipoles and according to the Pauli principle [10] [11] they repel each other. This goes so far that the formation of this incredible high concentration of excitons forces the whole crystal lattice to expand against the applied pressure. We show this in Fig. 5 where we measure the lattice constant (5a) (with strain gauges) and the expansion coefficient (5b) of the crystal in an isobar at 11.9 kbar. We observe that at about $230 \mathrm{~K}$ the lattice expands by $1.6 \%$ isostructurally, an enormous amount. The expansion coefficient becomes negative, of course. We even think that the expansion is of first order (dashed-dot line), but the point by point measurement cannot reproduce this exactly, because we go from the semimetallic state to the excitonic state.

We can estimate the maximal number of excitons with the help of Figure 5(a) and we observe that the lattice expansion occurs spontaneously when entering the excitonic phase. There must be an energy balance between the lattice energy causing the expansion and the electronic energy of the excitons. The energy balance can be described by the first equation in Figure 6. We take the lattice constant change from Figure 5(a) to go from $5.93 \AA$ to $6.03 \AA$, compute $\Delta \mathrm{l} / \mathrm{l}$ and $\Delta \mathrm{V} / \mathrm{V}$. We choose a pressure of $8.5 \mathrm{kbar}$ and an $\mathrm{E}_{\mathrm{B}}$ of $70 \mathrm{meV}$ and compute the number of excitons $\mathrm{n}_{\mathrm{ex}}=3.9 \times 10^{21} \mathrm{~cm}^{-3}$ (red field). We also can compute the number of Tm ions in the crystal in the fcc structure and it is $\mathrm{n}_{\mathrm{Tm}}=1.8 \times 10^{22}$ $\mathrm{cm}^{-3}$ (yellow field). In other words the exciton concentration is about $22 \%$ of the atomic density, an enormous amount of excitons. With the exciton orbit of $18 \AA$ it is quite clear that we have an exciton band or an exciton condensation. Since the exciton couples to a phonon the condensation is a Bose condensation, not a Bose-Einstein condensation. We can also estimate the Bose condensation temperature shown in Figure 6, where the general accepted formula yields $T_{B}=130$ $\mathrm{K}$ the right order of magnitude. The holes of the exciton are in a narrow $4 \mathrm{f}$-band and with a pressure change of 5 to $8 \mathrm{kbar}$ (Figure 4) one scans the width of the 4f-band [12]. The closing rate of the semiconductor rate has been measured to be $\mathrm{d} \Delta \mathrm{E} / \mathrm{dp}=-11 \mathrm{meV} / \mathrm{kbar}[4]$, so $3 \mathrm{kbar} \cdot 11 \mathrm{meV} / \mathrm{kbar}=33 \mathrm{meV}$ for the width of the narrow $4 \mathrm{f}$-band. From this in turn we use the general estimate that a band width of $1.5 \mathrm{eV}$ yields an effective mass of $\mathrm{m}_{\mathrm{e}}$ and derive that a band width of 30 $\mathrm{meV}$ corresponds to an effective hole mass $\mathrm{m}_{\mathrm{h}} \approx 50 \mathrm{~m}_{\mathrm{e}}$. The excitons are thus heavy bosons.

Here we want to make some remarks about this exciton condensation. Nobody in the world (to the best of our knowledge) has a comparable concentration of excitons which exist as long as we can sustain the pressure and as the 


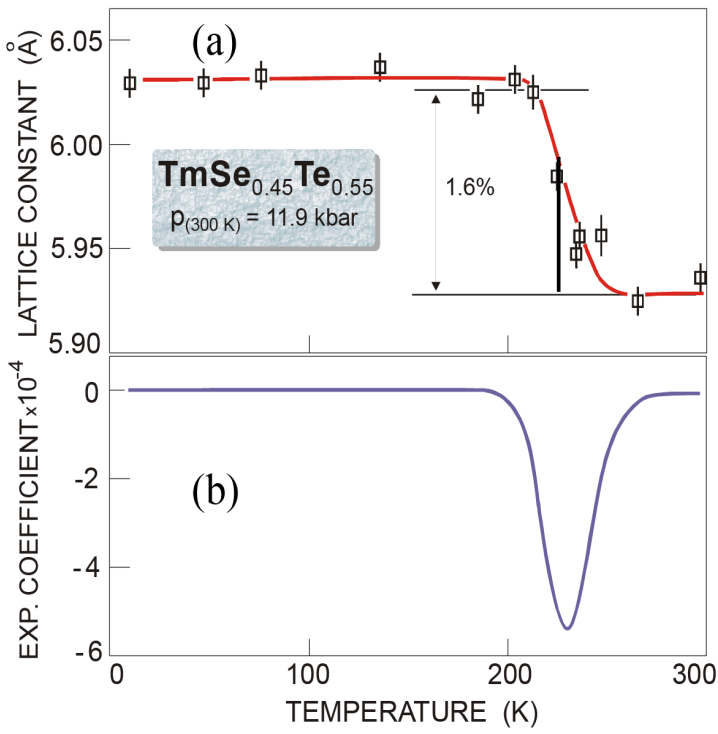

Figure 5. (a) (b) Isobar lattice constant and expansion coefficient of $\mathrm{TmSe}_{0.45} \mathrm{Te}_{0.55}$ [12].

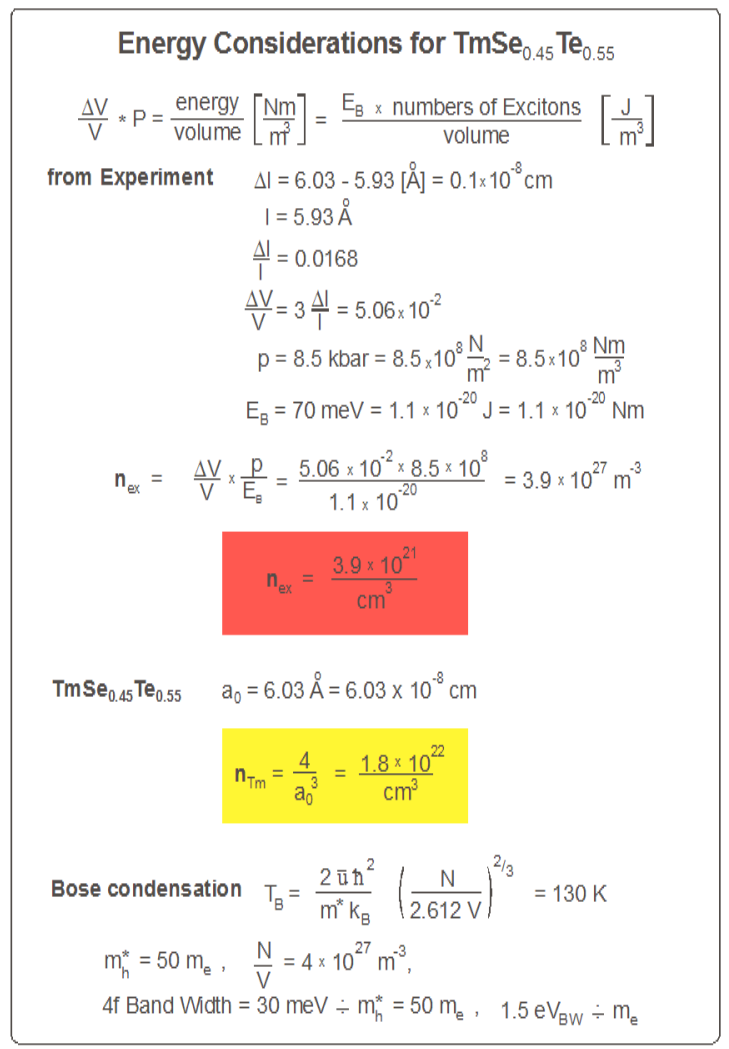

Figure 6. Calculations of the exciton concentration.

liquid Helium lasts, this means for days. We can make all kind of experiments in this condition, such as electrical conductivity, Hall effect, compressibility, heat conductivity, superfluidity, ultrasound velocity, phonon dispersion and specific heat. Nobody else has these possibilities. But the experiments are very demand- 
ing, low temperature and simultaneous pressure and doing the experiment which one wants to make.

\section{Phase Diagram of Semiconductor, Excitonic Insulator and Semimetal}

We plot in Figure 7 the coexistence ranges of the intermediate valence semiconductor, the excitonic insulator and the intermediate valence semimetal. We see that the highest temperature for which the excitonic insulator exists is about $260 \mathrm{~K}$ and the pressure range is between 7 and $13-14 \mathrm{kbar}$ (pressures applied at room temperature). Experimentally one can only measure isobars in a clamped pressure cell. However, the isobars in Figure 7 are no straight lines, because the pressure applied at room temperature relaxes somewhat at low temperatures. In the inset of Figure 7 we see the Hall effect, which measures the free electron concentration in the $5 \mathrm{~d}$ band. In the semimetallic state (curve M) at $13 \mathrm{kbar}$ the electron concentration is about $3 \times 10^{21} \mathrm{~cm}^{-3}$. For the excitonic insulator at 8 kbar the free electron concentration is about $10^{18} \mathrm{~cm}^{-3}$ because now the free electrons condense into the excitons and do not contribute anymore to the Hall effect. In fact we observe that the carrier concentration reduces by about 3 orders of magnitude, the same as has been observed in Figure 4 for the electrical resistivity. The change in resistivity is thus mainly an effect in the carrier concentration though the mobility changes also somewhat [13]. The concentration of the excitons is then $3 \times 10^{21} \mathrm{~cm}^{-3}-10^{18} \mathrm{~cm}^{-3}=3 \times 10^{21} \mathrm{~cm}^{-3}$, about the same as has been obtained in Figure 4.

We can consider in an analogy a pot with soup. The pot is the hard surrounding of the crystal and inside is a soup of liquid excitons.

In Figure 8 we show in the upper part a proposal from Walter Kohn [14] from 1968 with the 3 phases semiconductor, excitonic insulator and semimetal plotted against the energy gap $\Delta \mathrm{E}$ with increased pressure going to the left. When $\Delta \mathrm{E}=\mathrm{E}_{\mathrm{B}}$ the excitonic instability starts. In the lower part of the figure we show the E-k diagram again for the 3 phases. It is surprising and satisfying that the foresight of Walter Kohn has practically reached reality by comparing the inset of Figure 8 with the real phases of $\mathrm{TmSe}_{0.45} \mathrm{Te}_{0.55}$ as shown in Figure 7.

\section{Isotherm and Compressibility}

We may ask, what is direct evidence for the condensed excitonic state. Typical for any liquid is its incompressibility. We can, for instance at $1.5 \mathrm{~K}$ apply an increasing pressure to $\mathrm{TmSe}_{0.45} \mathrm{Te}_{0.55}$ and this is shown in Figure 9 [12]. At first we cool at zero pressure from $300 \mathrm{~K}$ to $1.5 \mathrm{~K}$ and volume and lattice constant decrease. Then we increase pressure and measure the lattice constant with elastic neutrons through the pressure cell. Of course lattice constant and volume decreases further, corresponding to a Birch-Mournaghan equation (red curve). This is a very time consuming experiment, because for each pressure change the pressure cell had to be heated to room temperature to change to a higher 


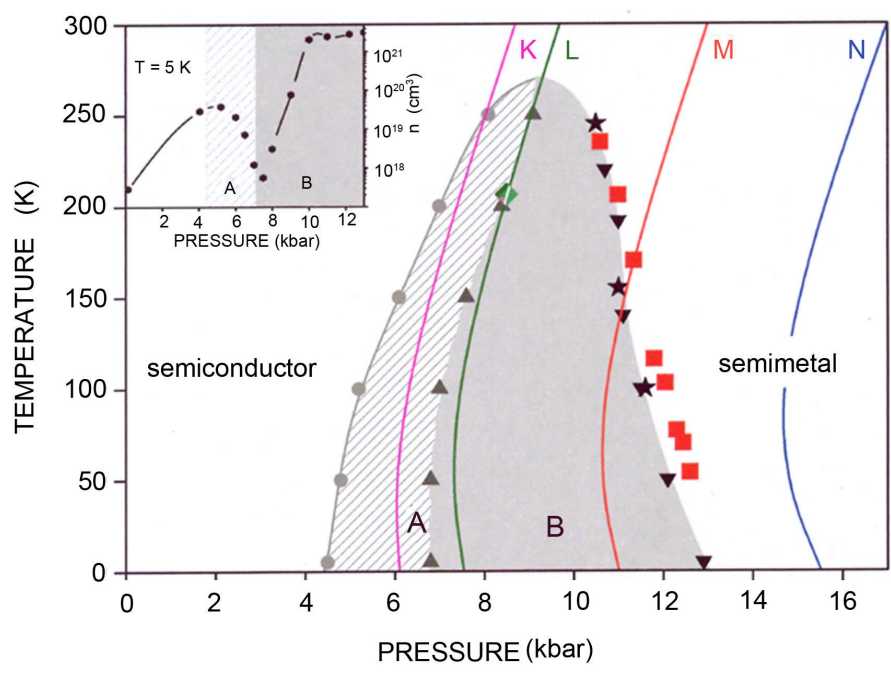

Figure 7. Temperature-pressure diagram of $\mathrm{TmSe}_{0.45} \mathrm{Te}_{0.55}$ with 3 regions: intermediate valence semiconductor, excitonic insulator (A, B), intermediate valence semimetal. The lines $\mathrm{K}, \mathrm{L}, \mathrm{M}, \mathrm{N}$ represent isobars, which are curved since the pressure applied at $300 \mathrm{~K}$ relaxes somewhat at low temperatures. The inset shows the $5 \mathrm{~d}$ free carrier concentration from a Hall effect in function of pressure and at $5 \mathrm{~K}$ [6] [13].

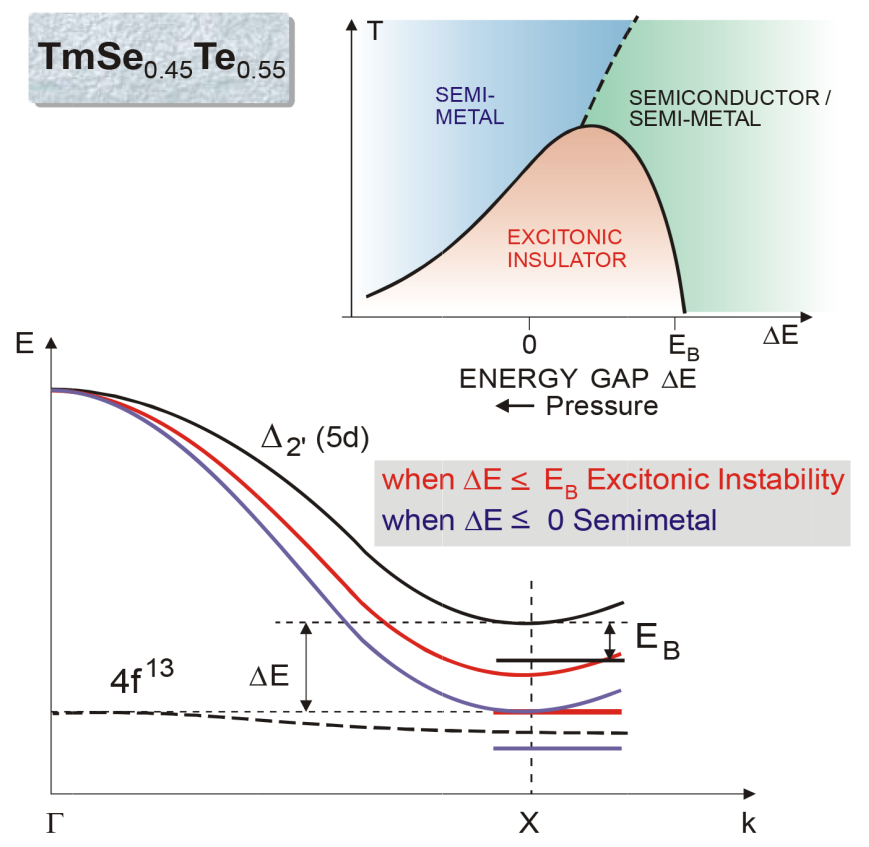

Figure 8. In the upper part we show a proposal by Walter Kohn [14] of the excitonic insulator, long before any experimental evidence. When $\Delta \mathrm{E}=\mathrm{E}_{\mathrm{B}}$ the excitonic instability starts. In the lower part we show again the $\mathrm{E}-\mathrm{k}$ diagram of $\mathrm{TmSe}_{0.45} \mathrm{Te}_{0.55}$ in the 3 phases.

pressure and then cooled down again and adjust the sample in the neutron beam and wait for beam time. Therefore this experiment has only 4 points, but at the relevant pressures. As can be seen in Figure 9 when entering the excitonic state 


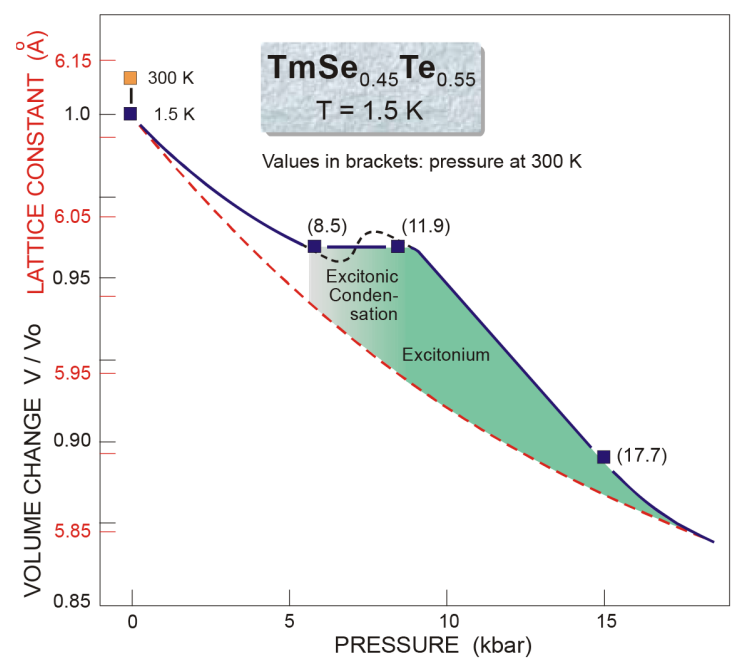

Figure 9. Isotherm of $\mathrm{TmSe}_{0.45} \mathrm{Te}_{0.55}$ at $1.5 \mathrm{~K}$ at relevant pressures. In brackets values at $300 \mathrm{~K}$ [12].

the lattice constant remains unchanged with increasing pressure, which means a compressibility of zero, as shown in Figure 10.

Taking experimental uncertainties into account we have at least a compressibility just as for diamond. Thus we can take this experiment as evidence of an excitonic liquid.

\section{Heat Conductivity and Superfluidity in the Excitonic Liquid}

We now want to discuss the possibility of superfluidity in the excitonic liquid. Here we resort at first to theory [11] [15]. There is a similarity between pairs of particles: 2 electrons can condense and produce superconductivity, an electron-hole pair (exciton) can upon condensation result in superfluidity. A positron pair should also result in superconductivity, but no such experiment is known.

In any case our exciton condensation may result in superfluidity. What would be the experiment to prove this? In our opinion this is heat conductivity [16], because it would diverge when the material becomes superfluid, just as in ${ }^{4} \mathrm{He}$ [17].

The experimental arrangement to measure heat conductivity and thermal diffusivity in a pressure cell is described in detail in Ref. [16], but the essence are isobars between $4 \mathrm{~K}$ and $300 \mathrm{~K}$ at various pressures. We show the results of measurements of the heat conductivity $\lambda$ with isobars at 4 different pressures, one in the semiconducting range (compare Figure 7) with $7 \mathrm{kbar}$, one in the semimetallic range at $15 \mathrm{kbar}$, both outside the excitonic region, and at two pressures 13 and $14 \mathrm{kbar}$ within the excitonic range. Temperature has been measured automatically for each degree. In Figure 11 we collect a few relevant formulae for the heat conductivity $\lambda$ and the thermal diffusivity a. We see that the heat conductivity depends on the specific heat $c_{\mathrm{v}}$ and $\mathrm{l}_{\mathrm{ph}}$ in direction $\mathrm{x}$, the mean free path for phonon scattering. In short, $1_{\text {ph }}$ will increase with decreasing 


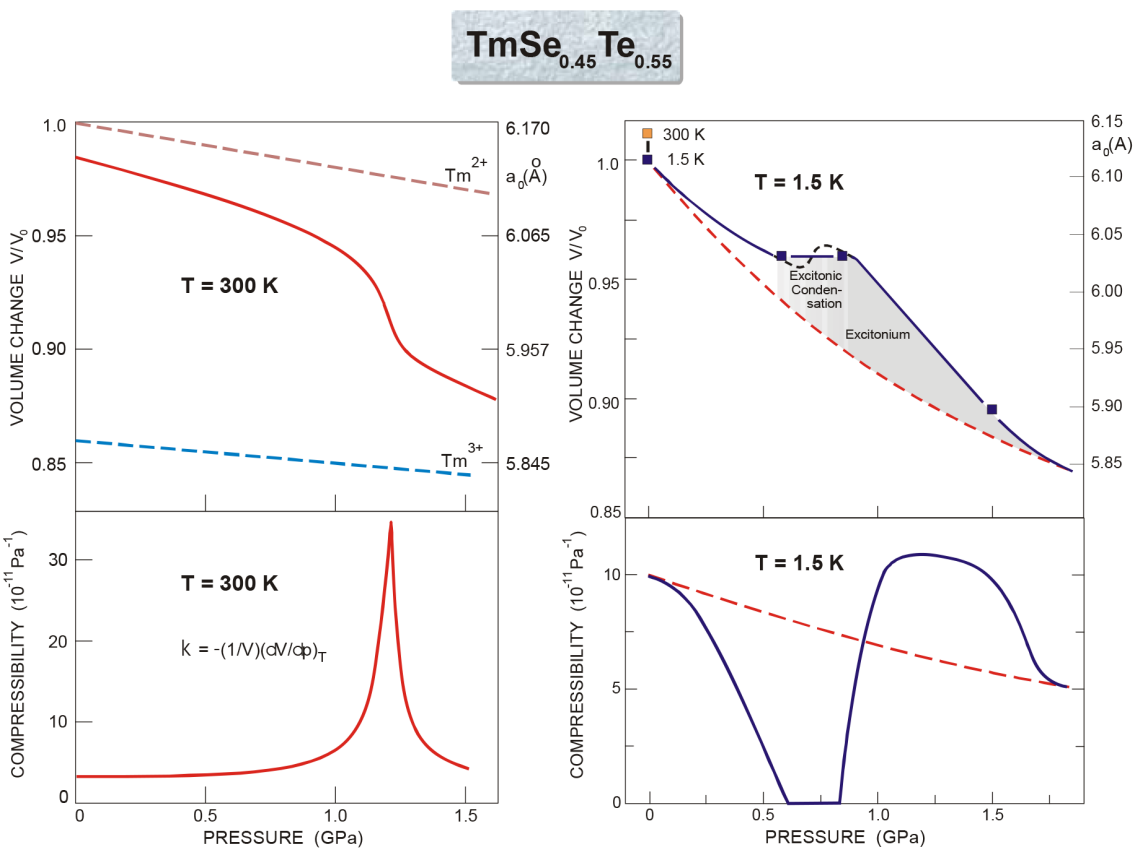

Figure 10. At room temperature (left hand figures) the volume change with pressure has a dramatic change near $11 \mathrm{kbar}$ at the transition semiconductor-semimetal, because the material is intermediate valence between $\mathrm{Tm}^{2+}$ and $\mathrm{Tm}^{3+}$ (also shown as reference in Fig. 10 ), it becomes soft with pressure. On the right hand side we show the material at $1.5 \mathrm{~K}$ and the compressibility goes to zero, the material becomes extremely hard, because we have now the exciton condensation.

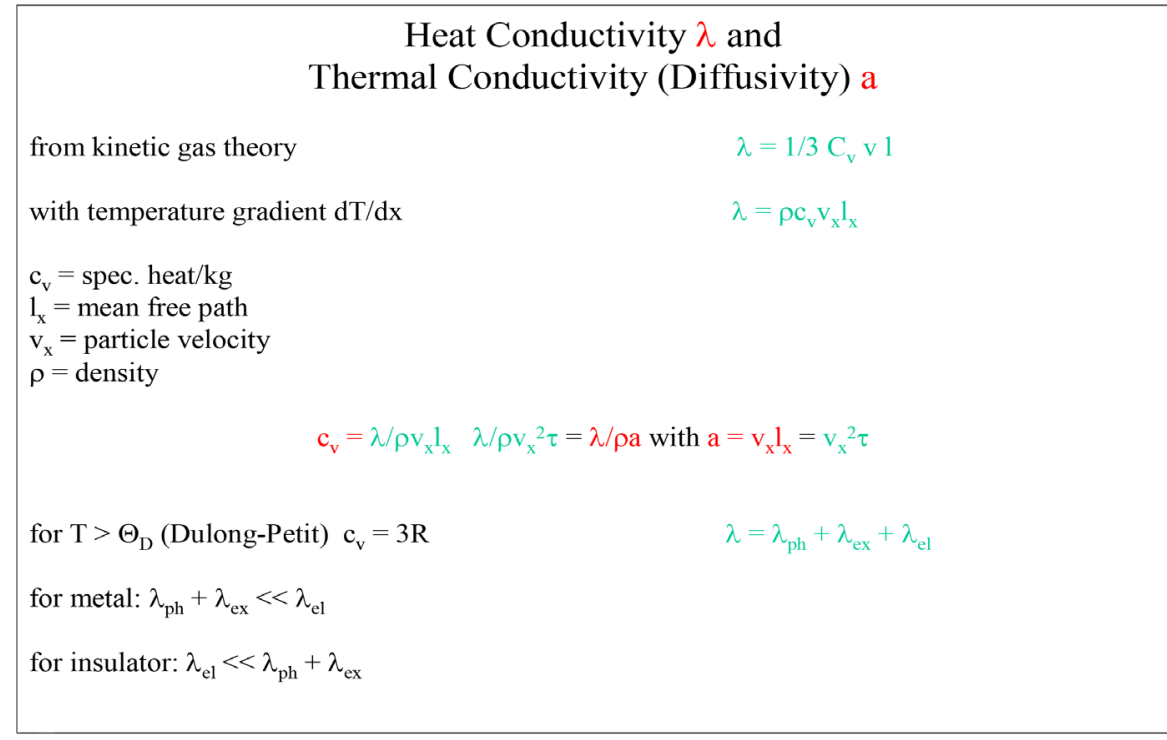

Figure 11. Formalities for the heat conductivity and the thermal diffusivity.

temperature because the density of phonons decreases and we have Umklapp processes involving 3 phonons. But the specific heat $c_{v}$ definitely will go towards zero for zero temperatures, thus the heat conductivity outside the excitonic region will display a maximum near $50 \mathrm{~K}$, as well for the semiconducting range (7 $\mathrm{kbar})$ as for the metallic range (15 kbar) and this is displayed in Figure 12 and 


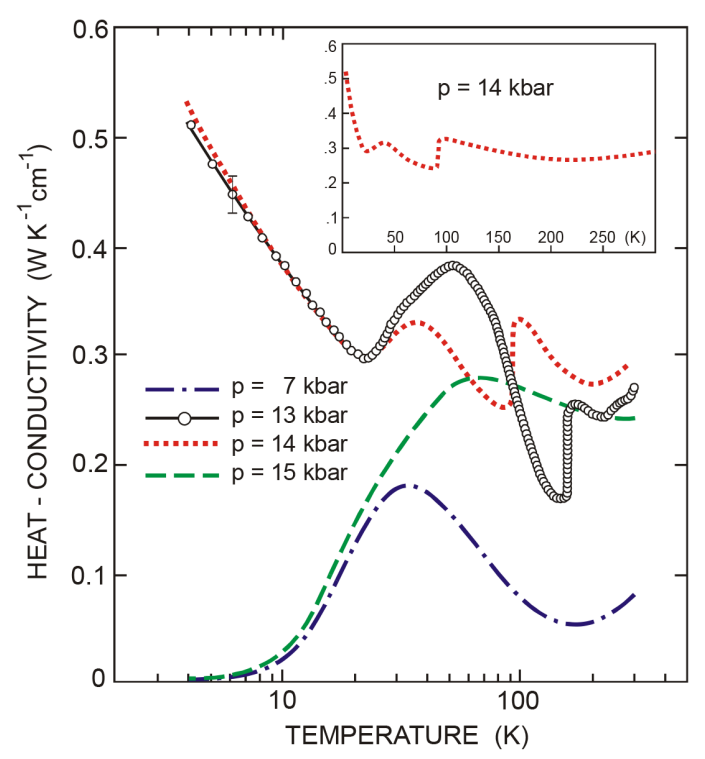

Figure 12. Heat conductivity $\lambda$ of $\mathrm{TmSe}_{0.45} \mathrm{Te}_{0.55}$ for various pressures in function of temperature. Dotted and full line in the excitonic region, dashed in the semimetallic region and dash-dotted line in the semiconducting phase. The inset shows the heat conductivity at 14 kbar in a linear scale [16].

this behavior is quite normal. The difference of the heat conductivity near $300 \mathrm{~K}$ for both cases is due to the electronic part of the heat conductivity in the metallic state and it corresponds roughly to the Wiedemann-Franz relation. This gives confidence to the measurements. We continue with the heat conductivity in the excitonic region at 13 and 14 kbar. We observe an unexpected downward jump in a first order transition when entering the excitonic phase. Consulting Figure 7 it is obvious that at different pressures one enters the excitonic phase at different temperatures. At these temperatures and pressures one enters the insulating excitonic phase mainly from the semimetallic phase, thus with a metal-insulator transition. The downward jumps in the heat conductivity $\lambda$ reflect the loss of the electronic part of the heat conductivity. The fascinating aspect of the heat conductivity in the excitonic region is the sharp increase of $\lambda$ below about $20 \mathrm{~K}$, quite in contrast with the $\lambda$ outside the excitonic region. Since $\lambda$ follows mainly the specific heat $c_{v}$ and the phonon mean free path $1_{\text {ph }}$, (Figure 11) and $c_{v}$ nevertheless must go to zero for $\mathrm{T} \rightarrow 0$, it is the phonon mean free path which goes faster to infinite than $c_{v}$ towards zero. Finally it means that the phonon mean free path becomes infinite. When one makes a heat pulse at one end of the crystal the excited phonon transports its energy without scattering on other phonons to the other side of the crystal, meaning an infinite heat conductivity. This is, however, only possible if the concentration of phonons as running waves is substantially reduced, because most of them couple to the heavy excitons as exciton-polarons, as we have seen before and thus more or less correspond to local modes. 
Unfortunately the measurements were limited to $4.2 \mathrm{~K}$, because at the time of the measurements one did not realize the implications. In any case $20 \mathrm{~K}$, the onset of the sharp increase of $\lambda$ with decreasing temperature can be considered as the onset of superfluidity, which, however, is different from the one of ${ }^{4} \mathrm{He}$, inasmuch as there the onset of superfluidity is a first order transition [17]. For our exciton case we propose a superthermal current in the two-fluid model, where the superfluid part increases gradually towards zero temperature [17]. A $\lambda$-anomaly in the specific heat as in the first order Bose-Einstein transition in ${ }^{4} \mathrm{He}$ is here not to be expected and also not found [17].

The proposed evidence of superfluidity within the condensed excitonic state necessitates an additional excitation spectrum of other quasiparticles, namely rotons or vortices [17]. $\lambda_{\text {tot }}$ is the sum of individual contributions (Figure 11) and below about $20 \mathrm{~K} \lambda_{\text {tot }}=\lambda_{\mathrm{ph}}+\lambda_{\mathrm{ex}} \cdot \lambda_{\mathrm{ph}}$ is the heat conductivity due to uncoupled phonons, which is proportional to $\mathrm{T}^{3}$ and can be neglected compared to $\lambda_{\mathrm{ex}}$ at low temperatures. Thus we obtain for $\lambda_{\mathrm{ex}}$ an Arrhenius law for the increase of the heat conductivity towards zero temperature $\lambda_{\text {ex }} \propto \exp \Delta / \mathrm{k}_{\mathrm{B}} \mathrm{T}$. This is shown in Figure 13 at $13 \mathrm{kbar}$. The activation energy or the gap $\Delta$ is $1 \mathrm{meV}$ or about $10 \mathrm{~K}$. The application of heat in the heat conductivity experiment can excite quasiparticles, e.g. rotons with gap energy of about $10 \mathrm{~K}$, which is the right order of magnitude. In superfluid ${ }^{4} \mathrm{He}$ the roton gap is $8.65 \mathrm{~K}$ [18].

\section{Thermal Diffusivity}

In Figure 14 we display the thermal diffusivity a for the same 4 pressures as in Figure 12. The thermal diffusivity $\mathrm{a}=\mathrm{v}_{\mathrm{x}} \mathrm{l}_{\mathrm{x}}$ and thus follows mainly the phonon mean free path $1_{x}$, with $v_{x}$ being about constant outside the excitonic region, consulting Figure 11. In fact outside the excitonic region with 7 and $15 \mathrm{kbar}$ it does exactly this, as can be seen in the theoretical curve for $1_{x}$ in Figure 15. For

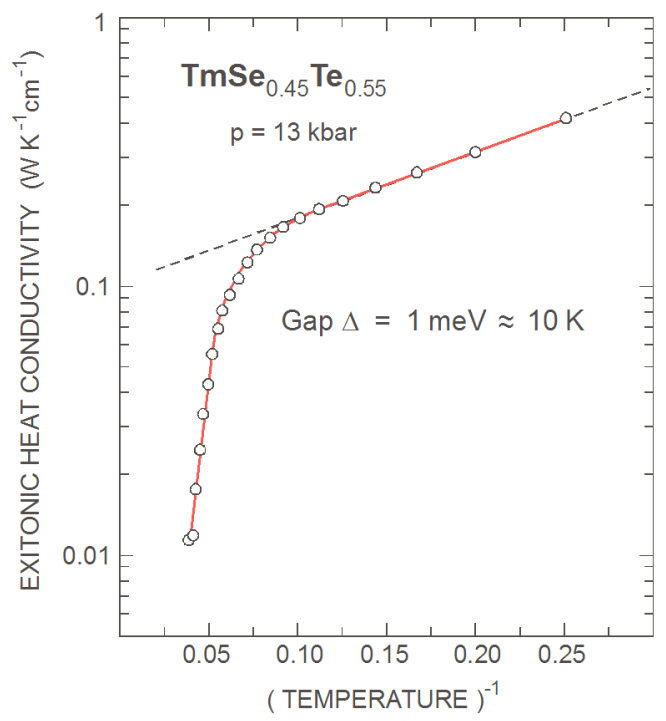

Figure 13. Excitonic part of the heat conductivity $\lambda_{\mathrm{ex}}$ at $13 \mathrm{kbar}$, shown in an Arrhenius plot [16]. 


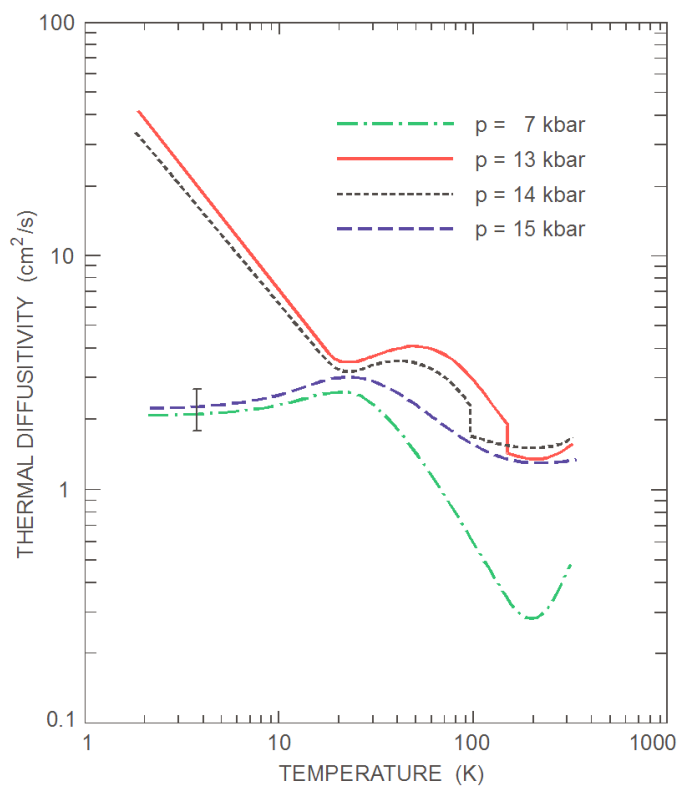

Figure 14. The thermal diffusivity in the semiconducting (7 kbar), semimetallic (15 kbar) and the excitonic phase (13 and $14 \mathrm{kbar}$ ) for $\mathrm{TmSe}_{0.45} \mathrm{Te}_{0.55}[16]$.

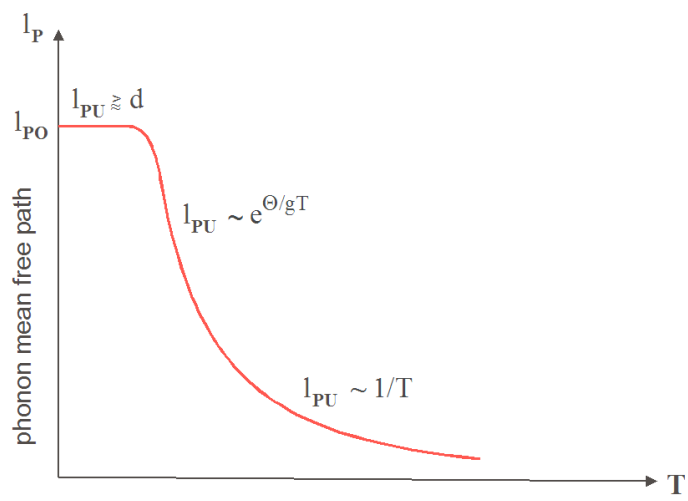

Figure 15. Theoretical curve for the mean free phonon path.

the lowest temperatures the phonon mean free path becomes the dimension of the crystal and is thus constant. But in the excitonic region, again below about 20 $\mathrm{K}$, the thermal diffusivity increases dramatically. Why then in the excitonic region the dimensions of the crystal do not seem to be important now? Just as in superfluid Helium heat can be transferred not only via phonon-phonon scattering in a diffuse manner, but ballistically via a highly directional quantum mechanical wave, the second sound. But also above $20 \mathrm{~K}$ there are anomalies, but they can be explained with the velocity of sound $\mathrm{v}_{\mathrm{x}}$, which increases now strongly in the excitonic region (see below).

In principle the two measurements of heat conductivity and thermal diffusivity permit the calculation of the specific heat $c_{v}=\lambda / \rho a$, with $\rho$ the density (see Figure 11) [16] and we did this in Figure 7 of Ref [16]. But we never felt very 
happy with this curve because we divided two point by point measurements. But the specific heat in the excitonic range is definitely below the one of the specific heat outside this range. But it is also very complex since the density $\rho$ diminishes when entering the excitonic phase, because the crystal expands (see Figure 5). It took us several years before we could make a direct measurement of the specific heat under pressure and below $300 \mathrm{~K}$ [6] [19].

\section{The Specific Heat}

The specific heat $c_{v}$ has been measured for $\mathrm{TmSe}_{0.45} \mathrm{Te}_{0.55}$ [6] [19] along isobars with $0 \mathrm{kbar}$, and corresponding to the curves $\mathrm{K}, \mathrm{N}, \mathrm{M}$ in Figure 7 , which is shown in Figure 16 [6] [19].

The molar specific heat $c_{\mathrm{m}}$ in J/mole K/f.u. has been matched at $300 \mathrm{~K}$ to the Dulong-Petit value of $52 \mathrm{~J} / \mathrm{mole} \mathrm{K} / \mathrm{f}$.u. The specific heat at ambient pressure represents a normal Debye curve (black curve). This curve has been measured by our colleagues at the university of Geneva and ETH Zürich for T $>1 \mathrm{~K}$ and $\mathrm{T}>$ $0.3 \mathrm{~K}$ [20]. A Schottky anomaly due to crystal field splitting of the Tm ions and an exchange splitting due to magnetic order at $0.23 \mathrm{~K}$ has been subtracted from the measured curve and the pure phonon contribution could be plotted as $c_{v} / T$ versus $\mathrm{T}^{2}$ and thus a Debye temperature $\theta$ of $117 \mathrm{~K}$ could be obtained [16]. Curve $\mathrm{N}$ is in the semimetallic high pressure phase outside the excitonic region and we find again a normal Debye curve (blue curve), but with a lower Debye temperature than at ambient pressure. This is at first sight surprising since at high pressure a solid becomes harder with a higher Debye temperature, but it has also been observed in Ref. [16]. A simple explanation can be that with high pressure we change somewhat the degree of valence mixing in the intermediate valence semimetal. Curve $\mathrm{M}$ (red curve) starts with about $13 \mathrm{kbar}$ at $300 \mathrm{~K}$ in the metallic region, but enters the excitonic region at about $150 \mathrm{~K}$. This occurs with a first order transition as we see in Figure 5(a). The specific heat should reveal a delta-function at the phase transition, but experimentally the spike reduces to a Gaussian shape [21]. All curves entering the excitonic region in Figure 7 from the semimetallic region in a first order transition (red squares and downward triangles in Figure 7) exhibit the Gaussian anomaly. We now discuss curve $\mathrm{K}$ (mauve), which enters the excitonic region in a second order transition without a spike in the specific heat. We observe that the specific heat is no longer a Debye curve, but below about $250 \mathrm{~K}$ (arrow in Figure 17) one finds a quasi linear drop of the specific heat until below about $30 \mathrm{~K}$ the specific heat joins the other curves. These measurements reveal a fundamental difference of the thermodynamic phases A and B in Figure 7.

In fact such a specific heat like curve $\mathrm{K}$ with a non-Debye like curvature has never been seen before. Since the specific heat over a higher and larger temperature interval is entirely given by the phonons (in the absence of magnetic order and special effects like Schottky anomalies), we must conclude a strong renormalization of the phonon spectrum. 


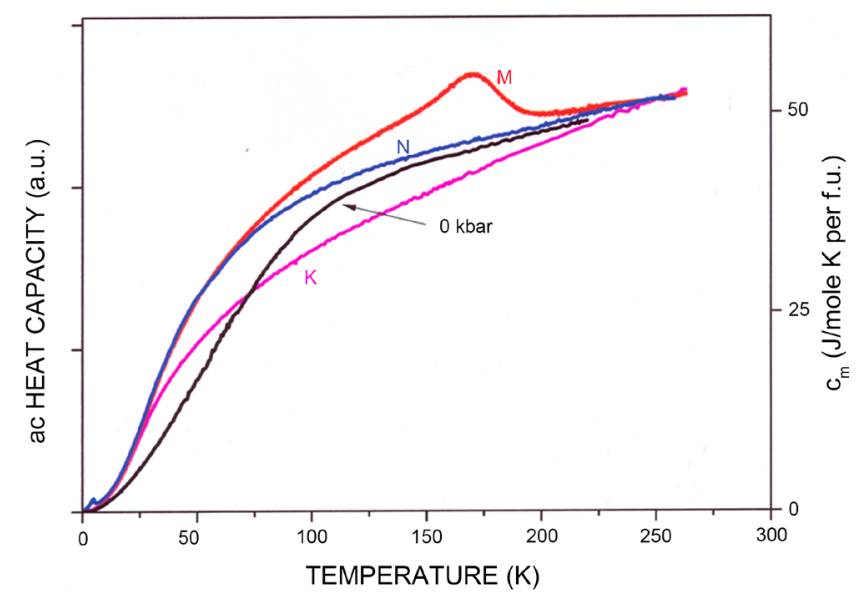

Figure 16. The measured specific heat of $\mathrm{TmSe}_{0.45} \mathrm{Te}_{0.55}$ at various pressures. The colors and letters are the same as in Figure 7 [6].

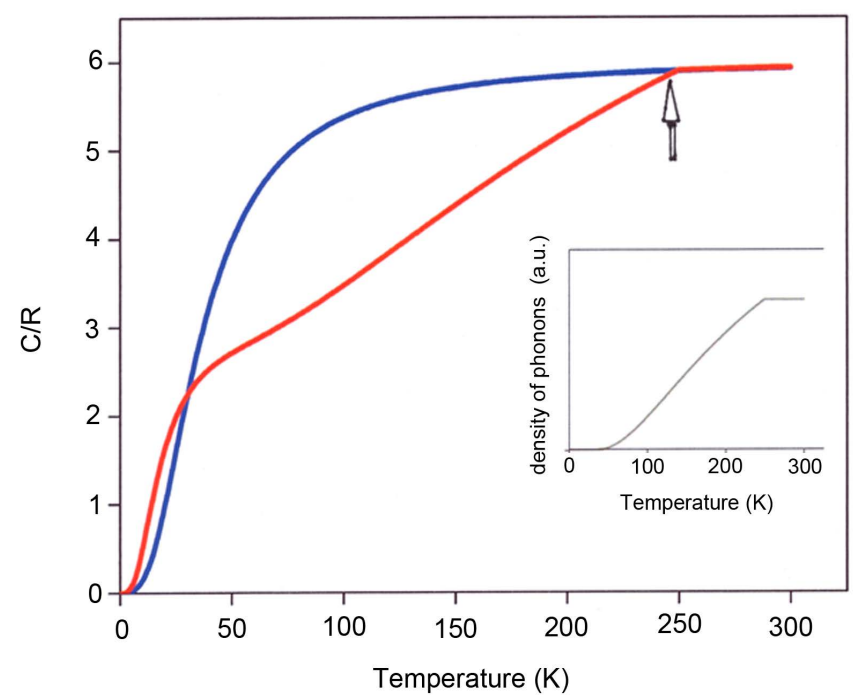

Figure 17. The specific heat of two typical schematic curves. One representing curve $\mathrm{N}$ in Figure 7 and typical for a Debye curve. The second representing curve $\mathrm{K}$ in Figure 7. The inset shows an assumed linear temperature dependence of the optical phonon density of states [6].

As we have stated already several times above the excitons in this indirect semiconductor couple strongly to phonons in a triple particle entity of hole-electron and phonon as an exciton polaron. But when the phonons couple to the heavy excitons with effective masses of the holes around $\mathrm{m}_{\mathrm{h}}=50 \mathrm{~m}_{\mathrm{e}}$ they become more or less localized like a local mode and do no longer contribute significantly to the specific heat. So an essential part of the Debye spectrum of the specific heat is missing.

So in Figure 17 we have made a model calculation of the specific heat with the assumption that the optical phonons are bound to the excitons below about 250 $\mathrm{K}$ (arrow in Figure 17) The acoustic branches of the phonons are modelled with 
a Debye and the optical phonons with an Einstein ansatz, respectively [6]. The seeming disappearance of phonons, i.e. the binding of the optical phonons on the excitons is represented with a linear decrease of the density of states below $250 \mathrm{~K}$ (inset to Figure 17). The model calculation in Figure 17 represents well the intriguing behaviour of curve $\mathrm{K}$ in Figure 16. A strong coupling regime for the phonons to ecxitons prevails and thus, while cooling, more and more wave like phonons become locked onto the excitons giving no more contribution to the specific heat. This renormalization of the phonon spectrum and the resulting effect on the specific heat has never been seen before and it is due to the extreme large concentration of exciton-polarons. Regarding now the Debye temperature $\theta$ of curve $\mathrm{K}$ in comparison with curve $\mathrm{N}$ we observe a further reduction of the Debye temperature, i.e. a minimum in the Debye temperature versus pressure in the excitonic region (red curve in Figure 17).

In a quantitative formula we can express

$$
c_{\exp }(T)=c_{a c}(T)+\rho_{o p t}(\omega, T) \frac{\left(\Theta_{E} / T\right)^{2} \mathrm{e}^{\left(\Theta_{E} / T\right)}}{\left(\mathrm{e}^{\left(\Theta_{E} / T\right)}-1\right)^{2}} .
$$

(Debye) + (Einstein) with $\rho_{\text {opt }}$ a temperature dependent density of optical phonons (see inset Figure 17). In fact the model calculation in Figure 17 represents quite well the measured specific heat of curve $\mathrm{K}$ in Figure 16. Thus the acoustic phonons alone exhibit a Dulong-Petit value c/R of $3 \mathrm{cal} /$ degree and the optical phonons have a temperature dependent density, their decrease with temperature representing the increase of excitons-polarons with decreasing temperature. The free optical phonons get lost for e.g. the thermal conductivity. However below about $20 \mathrm{~K}$ the excitons-polarons take over in the heat conductivity or the thermal diffusivity and with a diverging increase in these entities finally lead to superfluidity.

Curve $\mathrm{M}$ in Figure 16 is on the decreasing branch of exciton concentration (see Figure 7) where more and more free electrons in the $5 \mathrm{~d}$ band are screening the Coulomb interaction between electron and hole. In dissolving the excitons in region B of Figure 7 the electrons from the excitons enhance the $5 \mathrm{~d}$ electrons further which leads to a cumulative process and a collective breakdown of the rest excitons in a first order transition. Curve $\mathrm{M}$ enters the excitonic region at a temperature of about $150 \mathrm{~K}$ where the exciton concentrations are already about 2 orders of magnitude lower than at the maximum.

\section{Sound Velocity}

An ultrasound transducer has been glued to one end of the crystal and with a multiple echo from the other end of the crystal over the known length of the crystal the sound velocity could be obtained. This is shown in Figure 18 for various pressures. At zero pressure and at 7 and $18 \mathrm{kbar}$ the sound velocity is about $4000 \mathrm{~m} / \mathrm{s}$ and there is not much change with pressure. But best seen at $12 \mathrm{kbar}$, when entering the excitonic phase at $180 \mathrm{~K}$ the sound velocity is enhanced by nearly a factor 2 (see Figure 7). With $10 \mathrm{kbar}$ we are entering the excitonic 


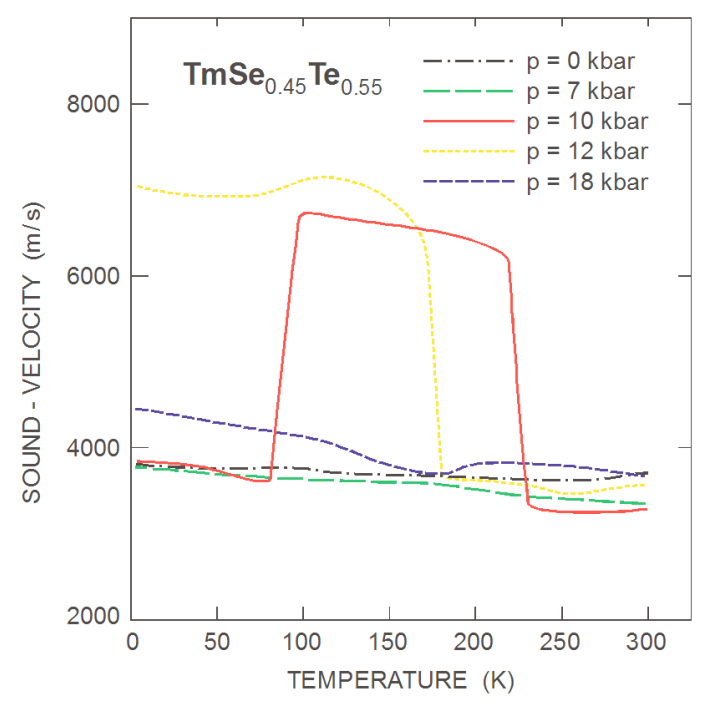

Figure 18. Longitudinal sound velocity outside and inside the excitonic region.

phase at $240 \mathrm{~K}$, again with a jump of nearly a factor 2 but near $90 \mathrm{~K}$ the pressure loss in the cell was just the size for a re-entrant transition to the non-excitonic phase. This was a unique phenomenon, but supporting the experimental measurements.

As mentioned above in the chapter about the thermal diffusivity a, the upwards jumps in the excitonic region are indeed caused by the jumps in the sound velocity. But not only this, the increase in sound velocity contributes directly to the thermal conductivity above $20 \mathrm{~K}$ and is responsible for the bumps in the thermal diffusivity.

The sound velocity is related to the bulk moduluss B and its inverse the compressibility. For a cubic material $B$ depends on the elastic moduli $c_{i j}$ as $B=1 / 3$ $\left(c_{11}+2 c_{12}\right)$ the elastic moduli instead, depend on the sound velocity as $c_{11}=$ $\rho \mathrm{v}_{\mathrm{L}[100]}^{2}$ and $\mathrm{c}_{12}=\rho\left(\mathrm{v}_{\mathrm{L}[100]}^{2}-2 \mathrm{v}_{\mathrm{T} 2[110]}^{2}\right)$. Assuming that in general $\mathrm{v}_{\mathrm{L}}$ is about 3 times $\mathrm{v}_{\mathrm{T} 2}$ we get the simplified relation $\mathrm{B} \approx \rho \mathrm{v}_{\mathrm{L}}^{2}$. thus in the excitonic phase we find a 2 times larger $\mathrm{v}_{\mathrm{L}}$ and thus a 4 times larger $\mathrm{B}$ or a 4 times smaller compressibility. The material gets indeed appreciable harder in the excitonic state.

In Figure 9 we have shown that between $5 \mathrm{kbar}$ and $8 \mathrm{kbar}$ the lattice constant remained practically constant during exciton condensation, meaning that the compressibility is close to zero. Putting a maximal error bar through the points of measurement a bulk modulus $\mathrm{B}=20 \mathrm{GPa}$ outside the excitonic region and a bulk modulus $\mathrm{B}=70 \mathrm{GPa}$ in the excitonic region could be obtained. From the sound velocity measurement in Figure 18 we calculate a bulk modulus $\mathrm{B}=24$ $\mathrm{GPa}$ outside the excitonic region and one of $100 \mathrm{GPa}$ in the excitonic region. So both types of measurements agree reasonable well and confirm the fact that during exciton condensation the material becomes extremely hard.

We offer two explanations for this phenomenon: the electron from the exciton enters a $5 \mathrm{~d}$-like orbit, which is much larger than the original $4 \mathrm{f}$ orbit it came 
from, and this in spite of the increasing pressure. Or the excitons, being electric dipoles, repel each other at short distances and large concentrations, creating a counter pressure to the applied pressure.

The dominant feature in Figure 18 is the sharp increase by a factor two of the sound velocity when entering the excitonic phase. But also at $300 \mathrm{~K}$ in an isotherm taken from Figure 18 with the relevant pressures the sound velocity is changing. Now at $300 \mathrm{~K}$ this is shown in Figure 19 but it has nothing to do with excitons and the change is much smaller than the one due to excitons. Here we find a minimum of the sound velocity with increasing pressure, inverse to what we have discussed in the excitonic region. The relation of bulk modulus B with sound velocity is $B \approx \rho v_{L}^{2}$ and a minimum of sound velocity implies a minimum in the bulk modulus which is inverse to the compressibility. Thus the minimum in the sound velocity means a maximum in the compressibility. This can be compared with the compressibility for $300 \mathrm{~K}$ in Figure 10 and we obtain a similar curve. At $300 \mathrm{~K}$ the softening of the bulk modulus or a maximum in the compressibility is here due to a change of the degree of valence mixing with pressure. So these completely different experiments (also by different authors [4]) support each other and give again confidence into the experiments.

\section{Dispersion of Exciton-Polarons}

We mentioned above regarding Figure 16 and Figure 17 that the Debye temperature in the excitonic region is less than the Debye temperature $\theta=117 \mathrm{~K}$ at $300 \mathrm{~K}$ but in Figure 18 we observe that the sound velocity is enhanced in the excitonic region. This seems to be a contradiction since in the Debye model the sound velocity is the slope of a linear phonon dispersion curve where the maximum frequency $\omega_{\max }$ determines the Debye temperature $\Theta$. A lower Debye temperature has thus a lower sound velocity and a lower bulk modulus. In order to explain a lower $\omega_{\max }$ together with a higher sound velocity we have to leave the simple Debye model for bare LA phonons $\omega \propto \sin (\mathrm{ka} / 2)$ and use a new dispersion curve of an exciton-polaron quasi-particle. This is no longer a simple sinus function. The dispersion of such an exciton-polaron is treated in textbooks, e.g. [22]. The result is that the phonon spectrum will be greatly renormalized in the excitonic region [23]. We show in Figure 20(a) LA phonons in $\Gamma$-X direction with $\omega_{\mathrm{LA}(\mathrm{I}-\mathrm{X})} \approx 14 \mathrm{meV}$ [12], in Figure $20(\mathrm{~b})$ an exciton with $4 \mathrm{f}$ character $\mathrm{E}_{\mathrm{B}}-\hbar^{2} \mathrm{k}^{2} / 2 \mathrm{M}$, where $\mathrm{M}$ is the sum of electron and hole mass of the exciton $\mathrm{M}=\mathrm{m}_{\mathrm{h}}+\mathrm{m}_{\mathrm{e}} \approx \mathrm{m}_{\mathrm{h}}$ with $\mathrm{m}_{\mathrm{h}} \approx 50 \mathrm{~m}_{\mathrm{e}}$.

We can see in Fig. 20 that now the dispersion of the exciton-polaron has indeed simultaneously a steeper slope (larger sound velocity) than the LA phonon and a lower $\omega_{\max }$ than the simple phonon (smaller Debye temperature).

\section{Theoretical Models}

Since the binding energy $E_{B}$ of the exciton-polaron is with $60-70 \mathrm{meV}$ relatively large also a Frenkel type of exciton-polaron is conceivable. Thus in a theoretical 


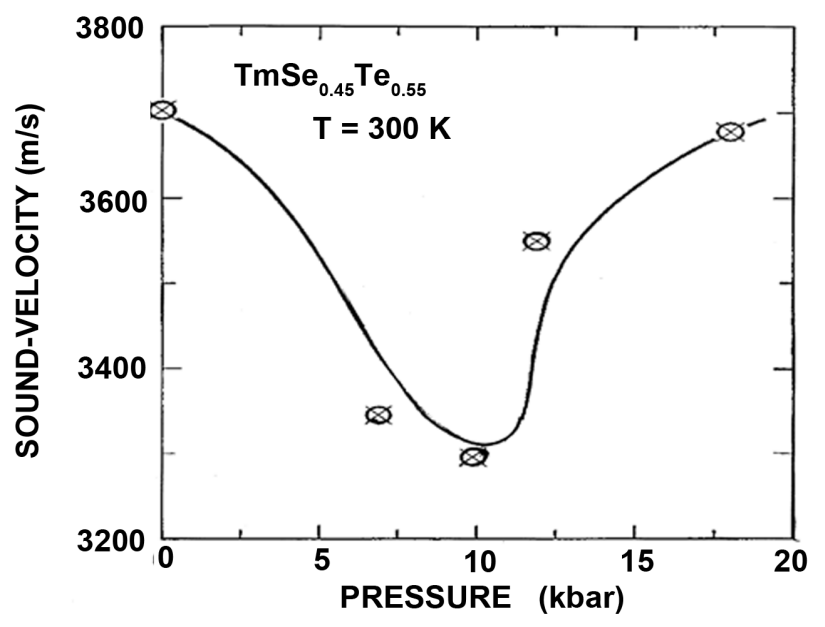

Figure 19. Sound velocity measurements at $300 \mathrm{~K}$ as a function of pressure [16].

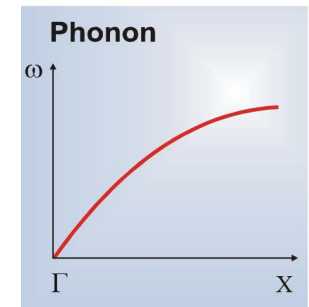

(a)

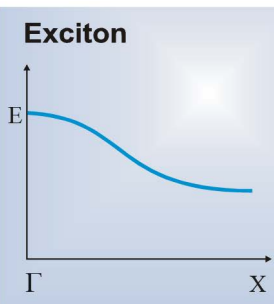

(b)

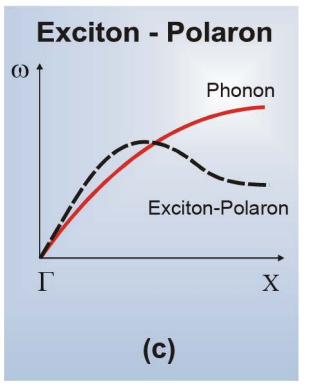

Figure 20. (a) Dispersion of a LA phonon; (b) Dispersion of a $4 \mathrm{f}$ exciton; (c) Dispersion of an exciton-polaron.

paper [24] it is proposed that the exciton condensation occurs in an extended Falikov-Kimball model [25] where, instead of the original model with localized $4 \mathrm{f}$ states a narrow hybridized $4 \mathrm{f}$ band is used, which is more realistic in this case. Extensively discussed has also been an effective mass model [26] with large differences between electron and hole mass, just as we proposed above. In a further paper [27] it has been shown, that weakly overlapping Frenkel type excitons can condense. Especially the coupling of excitons with phonons has been discussed in Ref. [28] and the formation of exciton-polarons. Finally in [29] exciton densities and superconductivity (sic) are discussed where for low exciton densities a Bose-Einstein condensate is proposed and for high density a Bardeen-Cooper-Schrieffer condensate should prevail, especially in coupled bilayers. This certainly is not the case in our experiments.

\section{Conclusions}

In this review paper we treat a special rare earth material, $\mathrm{TmSe}_{0.45} \mathrm{Te}_{0.55}$ which has been tailored so that with moderate pressures (up to $20 \mathrm{kbar}$ ) and low temperatures (down to $4 \mathrm{~K}$ ) an enormous amount of excitons $\left(10^{21} \mathrm{~cm}^{-3}\right)$, about $22 \%$ of the atomic density can be statically obtained. This high concentration of excitons with Bohr orbits of about $18 \AA$ leads to a condensation of excitons, in the 
sense of the excitonic insulator or excitonium which forms a liquid inside a crystalline surrounding. The existence range of condensed excitons is below 250 $\mathrm{K}$ and between 5 and $14 \mathrm{kbar}$. The condensation is accompanied with a phenomenon of incompressibility and as such with a compressibility near zero. In this condition the heat conductivity and the thermal diffusivity have been measured in order to investigate a possible superfluidity which has been proposed by Keldysh and Kopaev [11] and Kozlov and Maksimov [15]. Outside the excitonic region both entities behave quite normal, whereas in the excitonic region the heat conductivity diverges to ever increasing values. This can be explained below 20 $\mathrm{K}$ within a two-fluid model, where the superfluid part always increases until at temperature zero the complete condensed excitons become superfluid. The existence range of superfluidity thus has been increased from $2.3 \mathrm{~K}$ for ${ }^{4} \mathrm{He}$ by a factor of ten to $20 \mathrm{~K}$ for our material, which has never been seen before. Also the thermal diffusivity expands in the excitonic region above the phonon mean free path corresponding to the size of the crystal. This can be explained with the quantum-mechanical second sound, which is a ballistic transport of heat. The Debye temperature exhibits a minimum in the excitonic region, where nevertheless the sound velocity is increasing. These two incompatible measurements can be explained with a strong phonon renormalization in the excitonic region and the Debye phonon dispersion of LA phonons changes into the dispersion of an exciton-polaron, because every exciton binds to a phonon. This in turn means that the number of free phonons is strongly reduced in the excitonic region so that the specific heat becomes extremely anomalous, far away from a Debye specific heat. In general it can be said, that the anomalous physical properties of condensed excitons are unprecedented.

$\mathrm{TmSe}_{0.45} \mathrm{Te}_{\mathrm{o} .55}$ is not the only material where these phenomena can be observed. $\mathrm{YbO}$ and $\mathrm{YbS}$ are similar materials though one will need much larger pressures to close their gaps of about $1 \mathrm{eV}$ [30]. But also $\mathrm{Sm}_{0.75} \mathrm{La}_{0.25} \mathrm{~S}$ [31] is a possible candidate for which much lower pressures are needed. So with a good feeling for materials new and exciting effects can be found.

In a very new experiment on $1 \mathrm{~T}-\mathrm{TiSe}_{2}$ [32] the authors used momentum resolved electron energy loss spectroscopy (M-EELS) and observed that the electronic mode fell to zero at non zero momentum. They conclude that they observed evidence of the excitonic insulator, a part of our investigation. We published in Ref. 30 (1995), already a paper with the title "the discovery of excitonium", which is the excitonic insulator, which, however, the authors failed to quote.

\section{Acknowledgements}

The author wishes to thank Prof. Benno Bucher of HSR, Switzerland, for rechecking the experimental results and fruitful discussions.

\section{References}

[1] Mitani, T. and Koda, T. (1975) Wannier Excitons in the Ferromagnetic Semicon- 
ductors EuO and EuS. Physical Review B, 12, 2311. https://doi.org/10.1103/PhysRevB.12.2311

[2] Wachter, P., Kamba, S. and Grioni, M. (1998) Empty 4f States in TmS. Physica B, 252, 178-185. https://doi.org/10.1016/S0921-4526(98)00054-4

[3] Wachter, P. (1991) 19 Lanthanides/Actinides: Physics II, Intermediate Valence and Heavy Fermions. In: Gschneidner Jr., K.A., Eyring, L., Lander, G.H. and Chopin, G.R., Eds., Handbook on the Physics and Chemistry of Rare Earths, North Holland, Amsterdam, 177.

[4] Boppart, H. and Wachter, P. (1984) Pressure Induced Valence Transitions in the TmSe-TmTe System. Proc. Mater. Res. Soc., 22, 341.

[5] Neuenschwander, J. and Wachter, P. (1990) Pressure-Driven Semiconductor-Metal Transition in Intermediate-Valence $\mathrm{TmSe}_{1-\mathrm{x}} \mathrm{Te}_{\mathrm{x}}$ and the Concept of an Excitonic Insulator. Physical Review B, 41, 12693-12709. https://doi.org/10.1103/PhysRevB.41.12693

[6] Wachter, P. and Bucher, B. (2013) Exciton Condensation and Its Influence on the Specific Heat. Physica B, 408, 51-57. https://doi.org/10.1016/j.physb.2012.09.018

[7] Mott, N. (1961) The Transition to the Metallic State. Philosophical Magazine, 6, 287. https://doi.org/10.1080/14786436108243318

[8] Monnier, R., Rhyner, J., Rice, T.M. and Koelling, D.D. (1985) Electron-Hole Liquid as a True Ground State. Physical Review B, 31, 5554-5556.

https://doi.org/10.1103/PhysRevB.31.5554

[9] Wachter, P. (2015) Physical Properties of Some Stoichiometric Rare Earth Nitride Single Crystals. Advances in Materials Physics and Chemistry, 5, 96-131. https://doi.org/10.4236/ampc.2015.53013

[10] Halperin, B.I. and Rice, T.M. (1968) Possible Anomalies at a Semimetal: Semiconductor Transition. Reviews of Modern Physics, 40, 755-766. https://doi.org/10.1103/RevModPhys.40.755

[11] Keldysh, L.V. and Kopaev, A.N. (1965) Possible Instability of the Semimetallic State toward Coulomb Interaction. Soviet Physics, Solid State, 6, 2219-2224.

[12] Wachter, P. (2001) Exciton Condensation in an Intermediate Valence Compound: $\mathrm{TmSe}_{0.45} \mathrm{Te}_{0.55}$. Solid State Communications, 118, 645-650. https://doi.org/10.1016/S0038-1098(01)00202-2

[13] Bucher, B., Steiner, P. and Wachter, P. (1991) Excitonic Insulator Phase in $\mathrm{TmSe}_{0.45} \mathrm{Te}_{0.55}$. Physical Review Letters, 67, 2717-2720. https://doi.org/10.1103/PhysRevLett.67.2717

[14] Kohn, W. (1968) Many Body Physics. In: de Witt, C. and Balian, R., Eds., Gordon \& Breach, New York.

[15] Kozlov, A.N. and Maksimov, L.A. (1965) Metal-Dielectric Divalent Crystal Phase Transition. Journal of Experimental and Theoretical Physics, 21, 790.

[16] Wachter, P., Bucher, B. and Malar, J. (2004) Possibility of a Superfluid Phase in a Bose Condensed Excitonic State. Physical Review B, 69, Article ID: 094502. https://doi.org/10.1103/PhysRevB.69.094502

[17] Tilley, D.R. and Tilley, J. (1990) Superfluidity and Superconductivity. In: Brewer, D.F., Ed., Adam Hilger, Bristol and New York, 35.

[18] Henshaw, D.G. and Woods, A.D.W. (1961) Modes of Atomic Motions in Liquid Helium by Inelastic Scattering of Neutrons. Physical Review, 121, 1266-1274. https://doi.org/10.1103/PhysRev.121.1266 
[19] Bucher, B., Park, T., Thompson, J.D. and Wachter, P. (2013) Exciton Condensation and Its Influence on the Specific Heat. arXiv:0802.3354v2..

[20] The authors are most grateful to Prof. A. Junod (University of Geneva) and CH. Bergeman ETH Zürich for performing specific heat measurements at ambient pressure.

[21] Schilling, A., Fischer, R.A., Phillips, N.E., Welp, U., Kwok, W.K. and Crabtree, G.W. (1997) The Specific Heat in a Sample with a Temperature Gradient. Physical Review Letters, 78, 4833-4836. https://doi.org/10.1103/PhysRevLett.78.4833

[22] Hodgson, J.N. (1970) Optical Absorption and Dispersion in Solids. Butler and Tanner, London.

[23] Wachter, P. and Bucher, B. (2006) Superfluidity in Condensed Excitons below $20 \mathrm{~K}$. Physica Status Solidi, 3, 18-27.

[24] Ihle, D., Pfafferot, M., Burovski, E., Bronold, F.X. and Fehske, H. (2008) Bound State Formation and the Nature of the Excitonic Insulator Phase in the Extended Falicov-Kimball Model. Physical Review B, 78, Article ID: 193103.

https://doi.org/10.1103/PhysRevB.78.193103

[25] Falikov, L.M. and Kimball, J.C. (1969) Simple Model for Semiconductor-Metal Transitions: SmB6 and Transition-Metal Oxides. Physical Review Letters, 22, 997-999. https://doi.org/10.1103/PhysRevLett.22.997

[26] Bronold, F.X. and Fehske, H. (2006) Possibility of an Excitonic Insulator at the Semiconductor-Semimetal Transition. Physical Review B, 74, Article ID: 165107. https://doi.org/10.1103/PhysRevB.74.165107

[27] Zenker, B., Ihle, D., Bronold, F.X. and Fehske, H. (2012) Electron-Hole Pair Condensation at the Semimetal-Semiconductor Transition: A BCS-BEC Crossover Scenario. Physical Review B, 85, Article ID: 121102. https://doi.org/10.1103/PhysRevB.85.121102

[28] Zenker, B., Fehske, H. and Beck, H. (2014) Fate of the Excitonic Insulator in the Presence of Phonons. Physical Review B, 90, Article ID: 195118. https://doi.org/10.1103/PhysRevB.90.195118

[29] Soller, H. (2015) Exciton Condensates and Superconductors-Technical Differences and Physical Similarities. Journal of Applied Mathematics and Physics, 3, 1218-1225.

[30] Wachter, P. (1995) The Discovery of Excitonium. Journal of Alloys and Compounds, 225, 133-138. https://doi.org/10.1016/0925-8388(94)07023-7

[31] Wachter, P., Jung, A. and Pfuner, F. (2006) Exciton Condensation in Intermediate Valent $\mathrm{Sm}_{0.90} \mathrm{La}_{0.10}$ S. Physics Letters A, 359, 528-533. https://doi.org/10.1016/j.physleta.2006.06.073

[32] Kagar, A., Rak, M.S., Vig, S., Husain, A.A., Flicker, F., Joe, Y.J., Venema, L., MacDougal, G.J., Chiang, T.C., Fradkin, E., van Wezel, J. and Abbamonte, P. (2017) Momentum Resolved Electron Energy Loss Spectroscopy (M-EELS) in $1 \mathrm{~T}-\mathrm{TiSe}_{2}$. Science, 358, 1314-1317. 